EPJ manuscript No.

(will be inserted by the editor)

\title{
Transport coefficients in Chiral Perturbation Theory
}

\author{
D. Fernández-Fraile ${ }^{\mathrm{a}}$ and A. Gómez Nicola ${ }^{\mathrm{b}}$ \\ Departamentos de Física Teórica I y II, Universidad Complutense, 28040 Madrid, Spain.
}

Oct. 15,2006

\begin{abstract}
We present recent results on the calculation of transport coefficients for a pion gas at zero chemical potential in Chiral Perturbation Theory using Linear Response Theory. More precisely, we show the behavior of DC conductivity and shear viscosity at low temperatures. To compute transport coefficients, the standard power counting of ChPT has to be modified. The effects derived from imposing unitarity are also analyzed. As physical applications in Relativistic Heavy Ion Collisions, we show the relation of the DC conductivity to soft-photon production and phenomenological effects related to a nonzero shear viscosity. In addition, our values for the shear viscosity to entropy ratio satisfy the KSS bound.
\end{abstract}

PACS. 11.10.Wx Finite-temperature field theory - 12.39.Fe Chiral lagrangians - 25.75.-q Relativistic heavy-ion collisions

\section{Introduction}

We are interested in studying transport properties in a pion gas with zero baryon density. Pions are the lightest hadronic degrees of freedom and their dynamics is well described for low-enough energies and temperatures by Chiral Perturbation Theory (ChPT) 1]. ChPT is a lowenergy expansion performed in terms of $p^{2}$ ( $p$ represents a momentum, a mass or a temperature) against some scale $\Lambda_{\chi}^{2} \sim(1 \mathrm{GeV})^{2}$. However, as we shall see, the computation of transport coefficients in ChPT is intrinsically non-perturbative, leading to a modification of the standard ChPT power counting scheme to take into account collisions in the plasma (i.e. a non-zero pion width).

Considering small external perturbations which put the system slightly out of equilibrium, we can employ Linear Response Theory (LRT) 2,3,4, in which a transport coefficient $t$ is given by:

$$
\mathfrak{t}=C \lim _{q^{0} \rightarrow 0^{+}} \lim _{|\boldsymbol{q}| \rightarrow 0^{+}} \frac{\partial \rho\left(q^{0}, \boldsymbol{q}\right)}{\partial q^{0}},
$$

with $C$ a constant and $\rho$ the spectral density of a currentcurrent correlator.

In the calculation of transport coefficients, there is a dominant contribution coming from products of the kind $G_{\mathrm{A}} G_{\mathrm{R}} \propto 1 / \Gamma\left(G_{\mathrm{A}}\right.$ and $G_{\mathrm{R}}$ are the advanced and retarded propagators respectively), called pinching poles [3, 4, where $\Gamma$ is the particle width (which is perturbatively small: $\Gamma=\mathcal{O}\left(p^{5}\right)$ in ChPT [5]). From Kinetic Theory (KT), a behavior $\sim 1 / \Gamma$ for transport coefficients is also expected, since $1 / \Gamma$ represents the mean time between

\footnotetext{
a Electronic address: danfer@fis.ucm.es
}

b Electronic address: gomez@fis.ucm.es two collisions of the particles in the plasma. Each of these pinching poles comes from a pair of lines in a Feynman diagram contributing to the spectral density that share the same four-momentum when the limit in (11) is taken. Accordingly, there are some classes of diagrams potentially non-perturbative, being the most harmful the type called ladder diagrams (see Fig. 1). Another type, the so called bubble diagrams (see Fig. 2), which in principle would be the most harmful, for $n \geq 2$ turn out to give a negligible contribution to the electrical conductivity and the shear viscosity. Thus, the standard power counting scheme from ChPT has to be modified to compute transport coefficients 6 . In the case of a ladder diagram with $n$ rungs, its contribution is in principle of order $\mathcal{O}\left(p^{2 n} Y^{n+1}\right)$, where $Y$ is the contribution from a single-bubble diagram. For $T \ll M_{\pi} \simeq 140 \mathrm{MeV}$, a detailed analysis of the spectral function 6 shows that it contributes $\mathcal{O}\left(p^{2 n} Y\right)$. For $T \gtrsim M_{\pi}, Y$ becomes of order 1 or larger (due to unitarity effects) so that diagrams with one or more rungs could be non-negligible in this regime. Also note that, for high enough temperatures where typical momenta $p \sim T$, diagrams with derivative vertices become potentially dominant against those with constant vertices.

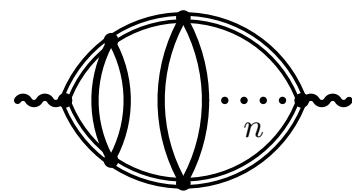

Fig. 1. Generic ladder diagram with $n$ rungs $(n \geq 1)$. Double lines represent particles with $\Gamma \neq 0$. 


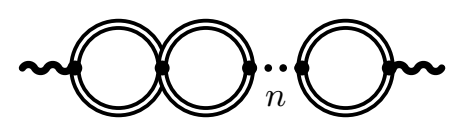

Fig. 2. Generic bubble diagram with $n$ bubbles $(n \geq 1)$. Double lines represent particles with $\Gamma \neq 0$.

\section{Electrical conductivity}

We are interested in the DC electrical conductivity, which represents the response of the gas under the action of a constant electric field. The expression for this transport coefficient in LRT is:

$$
\sigma=-\lim _{q^{0} \rightarrow 0^{+}} \lim _{|\boldsymbol{q}| \rightarrow 0^{+}} \frac{\rho_{\sigma}\left(q^{0}, \boldsymbol{q}\right)}{6 q^{0}},
$$

with

$$
\rho_{\sigma}\left(q^{0}, \boldsymbol{q}\right)=2 \operatorname{Imi} \int \mathrm{d}^{4} x \mathrm{e}^{\mathrm{i} q \cdot x} \theta(t)\left\langle\left[J_{i}(x), J^{i}(0)\right]\right\rangle,
$$

and $J^{i}$ is the electric current density in the gas. For this transport coefficient, at $T \ll M_{\pi}, Y \simeq \sqrt{M_{\pi} / T}$. So the contribution from a single-bubble diagram to the conductivity at these temperature is $\sigma^{(0)} \simeq e^{2} M_{\pi} \sqrt{M_{\pi} / T}$, where $e$ is the charge of the electron. The pion width is calculated in ChPT [5] from the pion-pion scattering amplitudes. To consider the unitarity effects on the transport coefficients, we impose unitarity on these amplitudes by means of the Inverse Amplitude Method (IAM) 7]. This unitarized scattering amplitudes have also been calculated at finite temperature [8]. We have implemented unitarization in the dilute gas approximation, where we neglect $\mathcal{O}\left(n_{B}^{2}\right)$ terms, with $n_{B}$ the Bose-Einstein distribution evaluated at a pion energy. This is approximately valid for $T \leq M_{\pi}$. In Fig. 3] we represent the behavior with temperature of the $\sigma^{(0)}$ contribution to conductivity. We see that unitarity makes conductivity grow near $T=170 \mathrm{MeV}$, as is expected in the QGP phase 9]. The contribution from ladder diagrams with derivative vertices has to be taken into account for temperatures $T \gtrsim M_{\pi}$.

The DC conductivity is related to the zero-energy (soft) photon spectrum emitted by the pion gas. Considering a pion gas produced after a Relativistic Heavy Ion Collision which expands cylindrically (Bjorken's hydrodynamical model), we obtain [6] the estimate $E \mathrm{~d} N_{\gamma} / \mathrm{d}^{3} \boldsymbol{p}\left(p_{T} \rightarrow\right.$ $\left.0^{+}\right) \simeq 5.6 \times 10^{2} \mathrm{GeV}^{-2}$ for the photon spectrum at zero transverse momentum. In Fig. 4 we see that this estimate is compatible with the experimental results obtained by the experiment WA98 [10] and other theoretical analysis 11. This is reasonable since it is expected that the hadronic contribution dominates at low $p_{T}$.

\section{Shear viscosity}

The expression for shear viscosity in LRT is

$$
\eta=\lim _{q^{0} \rightarrow 0^{+}} \lim _{|\boldsymbol{q}| \rightarrow 0^{+}} \frac{\rho_{\eta}\left(q^{0}, \boldsymbol{q}\right)}{20 q^{0}},
$$

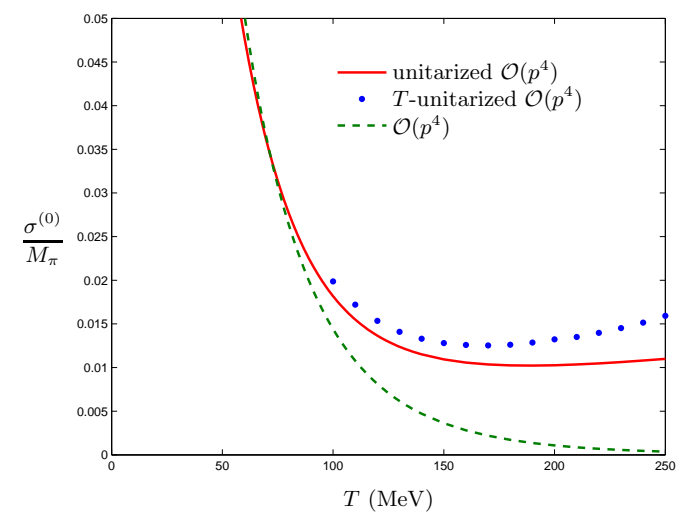

Fig. 3. Contribution to the electrical conductivity from the single-bubble diagram as a function of temperature. Three curves for the pion width are shown: non-unitarized $\mathcal{O}\left(p^{4}\right)$ (dashed), $T=0$ unitarized and finite $T$ unitarized.

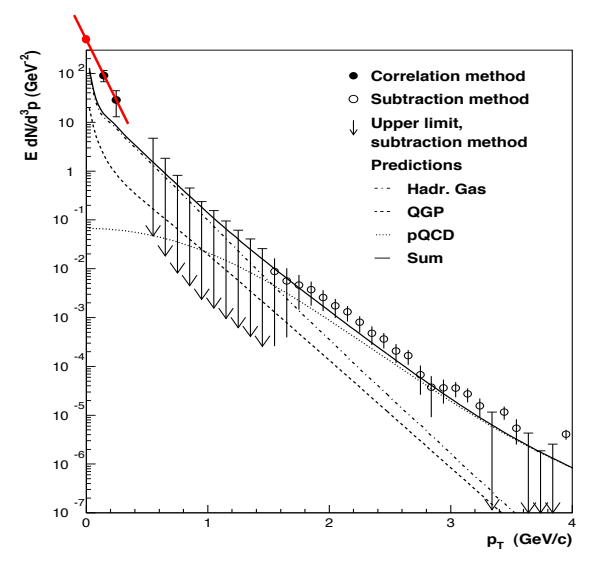

Fig. 4. Photon spectrum obtained by the experiment WA98 [10. We show the linear extrapolation from the two lowest energy points, which reaches a point at the origin compatible with our estimate. Note that theoretical predictions tend to underestimate the lowest energy points [1].

where

$$
\rho_{\eta}\left(q^{0}, \boldsymbol{q}\right)=2 \operatorname{Im} \mathrm{i} \int \mathrm{d}^{4} x \mathrm{e}^{\mathrm{i} q \cdot x} \theta(t)\left\langle\left[\pi_{i j}(x), \pi^{i j}(0)\right]\right\rangle,
$$

and $\pi_{i j}$ is the traceless part of the spatial energy-momentum tensor, i.e.

$$
\pi_{i j} \equiv T_{i j}-\frac{1}{3} g_{i j} T_{l}^{l}
$$

The shear viscosity has been previously calculated for a meson gas in the KT framework 12.13.14. For instance, in the work 12] (referred as DLE), a treatment based on a relativistic version of the Boltzmann equation is followed, using unitarized amplitudes from ChPT. In Fig. 5 we show our leading order result for $\eta$, where we see that unitarity also makes the shear viscosity change its behavior with $T$. Following the same steps as in [6], we readily get the behavior for $T \ll M_{\pi}$ as $\eta \simeq 37 F_{\pi}^{4} \sqrt{T} / M_{\pi}^{3 / 2}\left(F_{\pi} \simeq 93 \mathrm{MeV}\right.$ is the pion decay constant). This behavior is consistent with nonrelativistic KT 12 . 
In Fig. [5 we compare our leading order result with 12 , 13. The analysis in [12 is performed for fixed fugacity $z \equiv \mathrm{e}^{\beta\left(\mu_{\pi}-M_{\pi}\right)}$. We work at $\mu_{\pi}=0$, so that for the two values of $z$ shown in Fig. [5 we must compare at temperatures $T \simeq 30 \mathrm{MeV}$ (for $z=0.01$ ) and $T \simeq 200 \mathrm{MeV}$ (for $z=0.5)$. We get a reasonable agreement with 13 and a lower $\eta$ than 12 for $T>M_{\pi}$. The results in [14, where kaons are also included in the gas, also agree with ours for low and moderate temperatures. Nevertheless, our results should be taken with care, since for the shear viscosity, ladder diagrams with derivative vertices could become important already at $T \simeq 50 \mathrm{MeV}$.

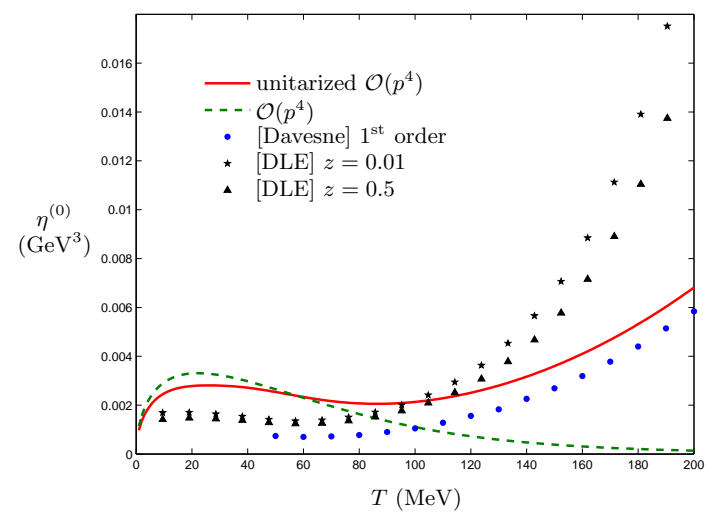

Fig. 5. Behavior of the unitarized shear viscosity with temperature and comparison with the results obtained in 1213 .

An interesting quantity is the ratio $\eta / s$, where $s$ is the entropy density. It has been conjectured [15] that the value $1 /(4 \pi)$ is a universal lower bound for this quantity in any physical system. On the other hand, the sound attenuation length $\Gamma_{s} \equiv 4 \eta /(3 s T)$ (neglecting bulk viscosity) enters directly in phenomenological effects observed in Relativistic Heavy Ion Collisions, such as elliptic flow or HBT radii [16. In Fig. [6] we plot this ratio for the pion gas. We see that in our analysis $\eta / s$ decreases monotonously, but it respects the KSS bound for temperatures up to the chiral phase transition $T_{c} \simeq 180-200 \mathrm{MeV}$. On general grounds, one expects $\eta / s$ to decrease below $T_{c}$ and increase logarithmically for very high temperatures 17 . The numerical values we get for $\Gamma_{s}(\simeq 1.1 \mathrm{fm}$ at $T=180 \mathrm{MeV})$ are in remarkable agreement with those used in [16] and at high $T$ our curve is not far from recent lattice and model estimates giving $\eta / s \sim 0.4-0.5$ above $T_{c}[18$.

In conclusion, we have presented a systematic method for evaluating transport coefficients in ChPT. The standard ChPT has to be modified, and as we go to higher temperatures, more diagrams have to be taken into account and eventually resummed. Even though the limitations of our approach are important, we get a reasonable agreement for physical quantities such as the very low $p_{T}$ photon spectrum or the viscosity to entropy ratio. We are currently analyzing the high-temperature diagrams, as well as the inclusion of kaons and other transport co-

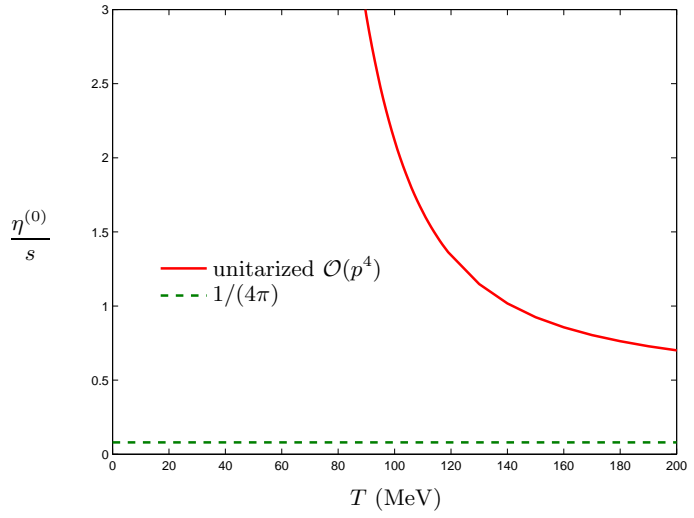

Fig. 6. Dependence of the $\eta / s$ ratio with temperature in ChPT. The entropy density $s$ is calculated to $\mathcal{O}\left(p^{5}\right)[19]$.

efficients such as bulk viscosity and thermal conductivity. Detailed results will be reported elsewhere.

We are grateful to A. Dobado and F. Llanes-Estrada for their useful comments. We also acknowledge financial support from the Spanish research projects FPA2004-02602, PR27/05-13955BSCH, FPA2005-02327 and FPI fellowship BES-2005-6726.

\section{References}

1. J. Gasser and H. Leutwyler, Ann. Phys. 158, (1984) 142.

2. M. Le Bellac, Thermal field theory (Cambridge Univ. Press, 2000).

3. S. Jeon, Phys. Rev. D 52, (1995) 3591-3642.

4. M. A. Valle Basagoiti, Phys. Rev. D 66, (2002) 045005.

5. J. L. Goity and H. Leutwyler, Phys. Lett. B 228, (2002) 517.

6. D. Fernández-Fraile and A. Gómez Nicola, Phys. Rev. D 73, (2006) 045025.

7. A. Gómez Nicola and J. R. Pelaez, Phys. Rev. D 65, (2002) 054009 .

8. A. Dobado, A. Gómez Nicola, F. J. Llanes-Estrada and J. R. Pelaez, Phys. Rev. C 66, (2002) 055201.

9. P. Arnold, G. D. Moore and L. G. Yaffe, JHEP 0011, (2000) 001.

10. M. M. Aggarwal et al. (WA98 Collaboration), Phys. Rev. Lett. 93, (2004) 022301.

11. S. Turbide, R. Rapp and C. Gale, Phys. Rev. C 69, (2004) 014903; W. Liu and R. Rapp, nucl-th/0604031.

12. A. Dobado and F. J. Llanes-Estrada, Phys. Rev. D 69, (2004) 116004.

13. D. Davesne, Phys. Rev. C 53, (1996) 3069-3084

14. M. Prakash, M. Prakash, R. Venugopalan and G. M. Welke, Phys. Rev. Lett. 70, (1993) 1228.

15. P. Kovtun, D. T. Son and A. O. Starinets, Phys. Rev. Lett. 94, (2005) 111601.

16. D. Teaney, Phys. Rev. D 68, (2003) 034913.

17. L. P. Csernai, J. I. Kapusta and L. D. McLerran, Phys. Rev. Lett. 97, (2006) 152303.

18. A. Nakamura and S. Sakai, Phys. Rev. Lett. 94, (2005) 72305; B. A. Gelman, E. V. Shuryak and I. Zahed, nucl-th/0601029.

19. P. Gerber and H. Leutwyler, Nucl. Phys. B 321, (1989) 387. 